\title{
Binary Particle Swarm Optimization for TDMA Broadcast Scheduling Problem
}

\author{
Sung-Soo Kim, Ji-Hwan Byeon \\ Dept. of Industrial Engineering \\ Kangwon National University \\ Chunchon, Korea \\ \{kimss, benjy86\}@kangwon.ac.kr
}

\author{
Hongbo Liu, Ajith Abraham \\ Machine Intelligence Research Labs \\ Auburn, Washington 98071, USA \\ lhb@dlut.edu.cn \\ ajith.abraham@ieee.org
}

\author{
Hong Yu \\ School of Computer Science \\ Dalian Ocean University \\ Dalian, China \\ yuhong_112@hotmail.com
}

\begin{abstract}
This paper presents binary particle swarm optimization (BPSO) for finding an optimum conflict-free transmission schedule for a broadcast radio network. This is known as Broadcast Scheduling Problem (BSP) and shown as an NP-complete problem in earlier studies. Because of this NP-complete nature, earlier studies used genetic algorithms, mean field annealing, neural networks, factor graph and sum product algorithm, and sequential vertex coloring algorithm to obtain the solution. We are able to achieve better results using our proposed BPSO. The results are obtained using our methodology is compared with CPLEX and all the other earlier solution methods.
\end{abstract}

Keywords-Broadcast scheduling problem (BSP); packet radio network, binary particle swarm optimization (BPSO); timedivision multiple access (TDMA); optimum transmission schedule

\section{INTRODUCTION}

The design problem that occurs in packet radio network is known as Broadcast Scheduling Problem (BSP). The objective of BSP is to find an optimal time-division multiple-access (TDMA) frame. The optimal TDMA frame should be able to schedule transmission of all nodes in a minimum TDMA frame length without conflict, and should also maximize the channel utilization or total conflict-free transmission. A TDMA frame is a time frame, which is divided into $M$ equal length time slots. In a TDMA cycle, each node should be scheduled at least once and is known as no-transmission constraint. Another constraint in this BSP scheduling problem is no-conflict constraint. No-conflict constraint has primary conflict and secondary conflict. Primary conflict constraint is that a node cannot transmit and receive simultaneously. Secondary conflict constraint is that a node is not allowed to receive more than one transmission simultaneously. With these constraints, the BSP problem is minimize $M$ (time slots) and maximize the channel utilization or total conflict-free transmission. Another objective is to minimize the average packet delay. The average packet delay is reduced if the channel utilization is maximized [1].

It is shown in earlier studies that this BSP is an NPcomplete combinatorial optimization problem. Hence, earlier studies used genetic algorithms, mean field annealing, neural networks, factor graph and sum product algorithm, sequential vertex coloring algorithm, and Simulated Annealing to obtain the solution. We will discuss the methodologies used in earlier studies.

Genetic Algorithms (GA) is a search algorithm based on the mechanism of natural selection that transforms a population (a set of individuals) into a new population (i.e., next generation) using genetic operators such as crossover, mutation and reproduction [2], [3], [4]. In the early works on GA [3], the solution (string) representation was restricted to only binary digits ( 0 and 1), and it is shown in [5] that a natural representation of strings are more efficient and produce better results. The applicability of GAs to broadcast scheduling problem is shown in [6]. In their study [6], a randomized algorithm is presented to create elite initial population. This randomized algorithm satisfies all the constraints of the BSP problem and so they obtain valid initial solutions. A modified crossover operator is also presented in [6], and a proof that all solution in every generation are valid solution; i.e., all solutions satisfy all the constraints.

The well-known Simulated Annealing (SA) is based on annealing process in statistical mechanics [7]. It is known that SA can be used for solving difficult combinatorial optimization problems. The broadcast scheduling problem is solved using the mean field analysis in [8]. Simulated annealing uses a stochastic process whereas Mean Field Annealing (MFA) uses a set of deterministic equations. Simulated annealing with valid solution mechanism is used for TDMA BSP [1].

The factor graph and the sum product algorithm presented in [9] is a selforganizing distributed algorithm using only local collaborative interactions among the neighboring nodes in the network. The approach used in [9] is divide the constraints of BSP into many simple rules, and these rules are enforced by a local processing unit in the factor graph. The optimal broadcast schedule is obtained by using the sum product algorithm in an iterative manner from the information exchanged among local processing units. A chaotic neural network for solving the BSP problem is given in [10].

Motivation of our study is to present a BPSO for TD- 
MA broadcast scheduling problem. First, we present the scheduling problem and our BPSO methodology in section II and III. In section IV, we present the results obtained using our methodology and CPLEX(IBM ILOG CPLEX optimization studio, optimization software package) and show that our method performs better than all other earlier solution methods, in terms of number of time slots used and utilization.

\section{The Broadcast Scheduling Problem}

In our previous work[1], we briefly describe the broadcast scheduling problem that arises in packet radio network considered in earlier studies [6], [9], [8], [10], [11], [12]. A given packet radio network can be represented by graph $G=(V, E)$, where vertices $V=1,2, \ldots, N$ are the radio network nodes and $N$ is the total number of nodes in the network. $E$ is the set of transmission links. In Broadcast Scheduling Problem (BSP), we need to obtain a schedule for transmission for all stations, so that no collision among packets takes place. In the schedule, the time is divided into slots of equal length. The number of time slots in each frame is fixed. We are interested in finding an optimal collision free transmission schedule for stations in a frame. Once this is obtained the frame is repeated in time. In the graph $G$, two nodes $i$ and $j(i, j \in E)$ are connected by an edge if and only if they can receive each other's transmission. In this situation, the nodes $i$ and $j$ are one hop away or adjacent neighbors. When two adjacent neighbors transmit in the same time slot, a primary conflict occurs. When a node receives two different packets from two of its adjacent neighbors in the same time slot, a secondary conflict occurs. The network topology can be represented by an $N \times N$ connectivity matrix $C$ defined as

$$
c_{i j}= \begin{cases}1 & \text { if nodes } i, j \text { are one hop apart } \\ 0 & \text { otherwise. }\end{cases}
$$

Another $N \times N$ matrix called compatibility matrix $D$ is defined as

$$
d_{i j}= \begin{cases}1 & \text { if nodes } i, j \text { are one hop apart or } \\ & \text { two hop apart } \\ 0 & \text { otherwise }\end{cases}
$$

Our objective is to obtain an optimal conflict free TDMA frame for packet transmission that also satisfies the constraints. This TDMA frame is repeated over time.

We use an $N \times N$ binary matrix $X=x_{m j}$ to denote a TDMA frame, where $M$ is the number of time slots in the TDMA frame. The matrix $X$ is defined as

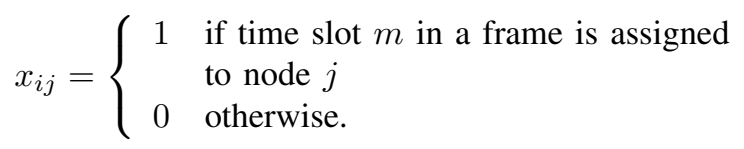

For node $j$ the channel utilization $\rho_{j}$ is defined as

$$
\begin{aligned}
\rho_{j} & =\frac{\text { the number of slots assigned to node } j}{\text { TDMA cycle length }} \\
& =\frac{\sum_{i=1}^{M} x_{m j}}{M}
\end{aligned}
$$

The channel utilization for the entire network $\rho_{j}$ is given as

$$
\rho=\frac{1}{M \times N} \sum_{m=1}^{M} \sum_{j=1}^{N} x_{m j}
$$

The objective of the BSP is to find a transmission (TDMA) frame with minimum number of time slots, which satisfies all the constraints and also maximize the channel utilization $\rho$. The BSP is described as:

BSP: Minimize $M$ and maximize $\rho$, subject to the following constraints

$$
\begin{gathered}
\sum_{m=1}^{M} x_{m j} \geq 1, \forall j \\
\sum_{m=1}^{M} \sum_{i=1}^{N} \sum_{j=1}^{N} x_{m i} \times x_{m j} \times d_{i j}
\end{gathered}
$$

Equation (6) represents the no-transmission constraint and equation (7) represents the no-conflict constraint.

\section{BinARY PARTICLE SWARM Optimization}

Difficult combinatorial optimization problems have been solved in a successful manner by using discrete particle swarm optimization [13]. The flowchart of binary particle swarm optimization (BPSO) for TDMA broadcast scheduling problem (BSP) in this paper is given in Fig. 1. For the BSP, the BPSO starts with a non-optimal (but a valid) initial TDMA schedule (solution). Initial valid and non-optimal TDMA schedule can be generated randomly. BPSO improves the initial TDMA schedule (solution) by selecting a new particle (TDMA schedule) which improves the objective function value.

In BPSO, we first select the initial valid solution (valid TDMA schedule) by using the randomized algorithm given in [6]. This initial valid solution (TDMA schedule) is further modified to improve the utilization $(\rho)$ and is used as a starting solution in BPSO. We call the modification as valid solution mechanism [1]. The modifications are done by using the information given by compatibility matrix ( $D$-matrix).

The valid solution mechanism [1] for improving the solution (TDMA schedule) is used in our study. In the BPSO methodology, the generation of initial feasible solutions has the following steps.

1) Decide the sequence to allot time slots for different nodes in the permutation sequence. 


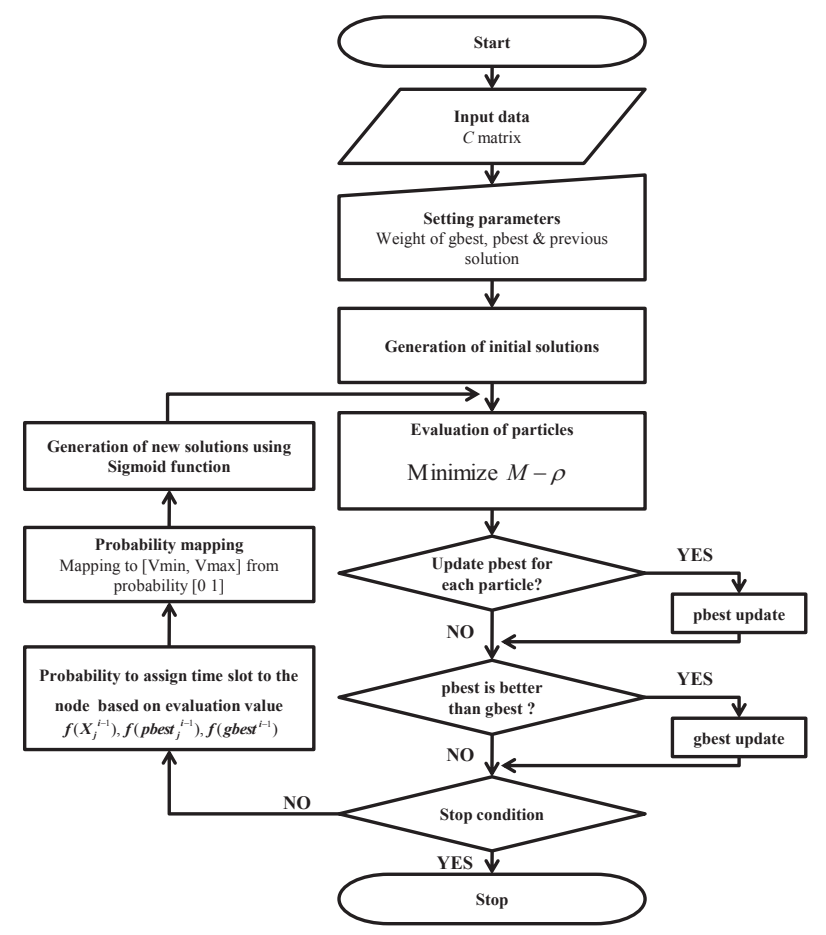

Figure 1. Flow-chart binary particle swarm optimization (BPSO)

2) Assign time slot 1 to the first node for the sequence.

3) Check that time slot 1 can be assigned to the second node using matrix $D$.

4) Assign time slot 2 to the second node if time slot 1 cannot be assigned to the second node. We assume that time slot 2 is assigned to the second node.

5) Check that time slot 1 and 2 can be assigned to the third node using matrix $D$.

6) Assign time slot 3 to the third node if time slot 1 and 2 cannot be assigned to the third node.

7) Follow the previous steps until all nodes are assigned to minimize the time slot $M$ if possible.

8) Maximize time slot utilization if possible using matrix $D$ after all nodes are assigned.

In the BPSO methodology, we can show the generation of new particles (solutions) using the following example.

$X_{j}^{i}=j$ th particle(solution) for generation $i$

$f\left(X_{j}^{i}\right)=$ Evaluation value of particle $X_{j}^{i}$

$i=$ Number of generation $(0<i<$ Maximum number of generation)

$j=$ Number of particle $(0<j<$ Number of particle $)$

As shown in Fig. 2,

$X_{1}^{1}$ is 1 st particle for generation 1

$X_{1}^{2}$ is 1 st particle for generation 2

pbest $_{1}^{1}$ is assigned the value of the particle 1 with best performance

gbest $^{1}$ is assigned the value of the particle with the best

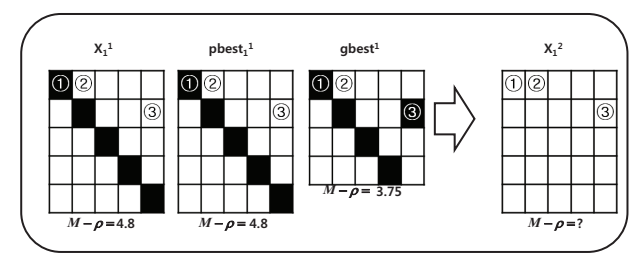

Figure 2. Generation of new particle $X_{1}^{2}$

performance so far in the all particles

We should minimize the value of $M-\rho$. The evaluation values of $X_{1}^{1}$, pbest $t_{1}^{1}$, gbest ${ }^{1}$ are assumed to be $4.8,4.8$, and 3.75. The weights of $X_{1}^{1}$, pbest $t_{1}^{1}$, gbest $^{1}$ are $w, r_{1} \times c_{1}$ , and $r_{2} \times c_{2}\left(w=1, c_{1}=2, c_{2}=3, r_{1}=0.8, r_{2}=0.6\right)$.

The probabilities at (1) $(1,1)$, (2) $(1,2)$, (3) $(2,5)$ of $X_{1}^{1}$, pbest $_{1}^{1}$, gbest $^{1}$ are 1 based on the following.

$$
\begin{aligned}
& \text { (1) : } \frac{\frac{1}{4.8} \times 1+0.8 \times \frac{1}{4.8} \times 2+0.6 \times \frac{1}{3.75} \times 3}{\frac{1}{4.8} \times 1+0.8 \times \frac{1}{4.8} \times 2+0.6 \times \frac{1}{3.75} \times 3}=1 \\
& \text { (2): } \frac{0}{\frac{1}{4.8} \times 1+0.8 \times \frac{1}{4.8} \times 2+0.6 \times \frac{1}{3.75} \times 3}=0 \\
& \text { (3): } \frac{0.6 \times \frac{1}{3.75} \times 3}{\frac{1}{4.8} \times 1+0.8 \times \frac{1}{4.8} \times 2+0.6 \times \frac{1}{3.75} \times 3} \\
& =0.4698
\end{aligned}
$$

The probabilities 1,0 , and 0.4698 are mapping to $v_{\min }=$ -4 and $v_{\max }=4$.

$$
\begin{aligned}
& v_{1}(t+1)=1 \times 8-4=4 \\
& v_{(2)}(t+1)=0 \times 8-4=-4 \\
& v_{3}(t+1)=0.4698 \times 8-4=-0.2416
\end{aligned}
$$

The Sigmoid function is used to calculate these probabilities.

$$
\begin{aligned}
& P_{(1)}=S\left(v_{(1)}(t+1)\right)=\frac{1}{1+e^{-4}}=0.9820 \\
& P_{(2)}=S\left(v_{(2)}(t+1)\right)=\frac{1}{1+e^{4}}=0.0180 \\
& P_{(3)}=S\left(v_{(3)}(t+1)\right)=\frac{1}{1+e^{0.2416}}=0.4399
\end{aligned}
$$

The time slot 1 of $X_{1}^{2}$ can be assigned to node 1 with probability $98.2 \%$ at the position (1). If the random number $(0 \sim 1)$ is less than 0.982 , then the time slot 1 for node 1 is assigned. Otherwise, the time slot 1 for node 1 is not assigned. The time slot 1 of $X_{1}^{2}$ can be assigned to node 2 with probability $1.8 \%$ at the position (2). If the random number $(0 \sim 1)$ is less than 0.0180 , then the time slot 1 for node 2 is assigned. Otherwise, the time slot 1 for node 2 is not assigned. The time slot 2 of $X_{1}^{2}$ can be assigned to node 5 with probability $43.99 \%$ at the position (3). If the random 


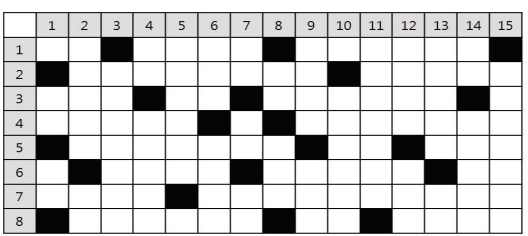

Figure 3. The optimal solution using our proposed method for 15 node network

Table I

COMPARISON OF OUR RESULTS FOR $N=15$ NODE NETWORK: BASED ON NUMBER OF TIME SLOTS $M$ AND UTILIZATION $\rho$

\begin{tabular}{lcl}
\hline \multirow{2}{*}{ Algorithm } & \multicolumn{2}{c}{ Node } \\
\cline { 2 - 3 } & \multicolumn{2}{c}{15} \\
\cline { 2 - 3 } & $M$ & $\rho$ \\
\hline CPLEX & 8 & 0.167 \\
Proposed method & 8 & 0.167 \\
Chen[9] & 8 & 0.167 \\
Kim[1] & 8 & 0.167 \\
Gunasekaran[14] & 8 & 0.167 \\
Wang[8] & 8 & 0.15 \\
Wang[10] & 8 & N/A \\
Wu[11] & 8 & 0.167 \\
Yeo[12] & 8 & 0.15 \\
\hline
\end{tabular}

number $(0 \sim 1)$ is less than 0.4399 , then the time slot 2 for node 5 is assigned. Otherwise, the time slot 2 for node 5 is not assigned.

\section{SimUlation RESULTS AND COMPARISON WITH OTHER METHODS}

In this section, we present the simulation results obtained by using our BPSO, and also present a comparison of performance of our BPSO with the results obtained in earlier methods. As mentioned in [6], there is no standard benchmark problem set and so we compare our results with the available results given in earlier studies, and show the performance improvement in packet radio networks using our BPSO methodology. The performance criteria used for comparison are $M$ (time slots), $\rho$ (channel utilization for the whole network), and the average time delay. We first consider a small-scale network with $N=15$. The TDMA schedule obtained using our BPSO methodology is shown in Fig. 3. In this Fig. 3, a black box represents an assigned time slot. The schedule obtained in our methodology has 8 $(M)$ time slots and the utilization of the whole network $(\rho)$ is $0.167(20 / 120)$ which is the same to the optimal solution using CPLEX. The same result is obtained in earlier studies [1], [9], [11], [14]. In other earlier studies [8], [12], the value of $M$ obtained is the same as our result i.e., $M=8$, but the utilization obtained is 0.15 which is less than the result obtained in our methodology. These results are shown in Table I.

We next consider a medium-scale network with $N=30$ and 40 . The best and optimal TDMA schedules obtained

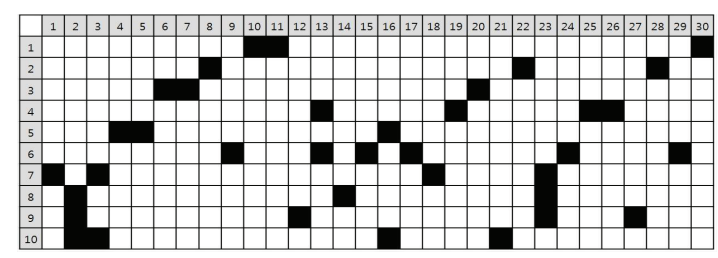

Figure 4. The optimal solution using our proposed method for 30 node network

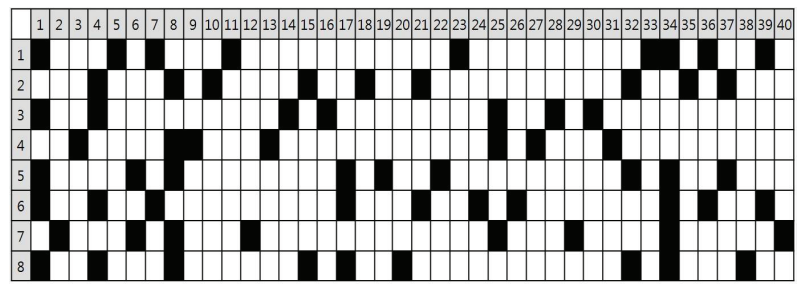

(a) The best solution using our proposed method

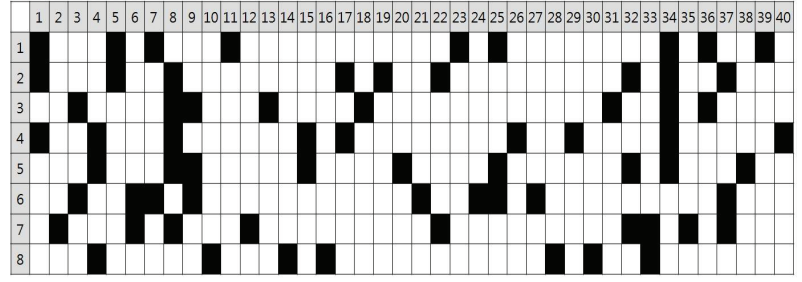

(b) The optimal solution using CPLEX

Figure 5. Comparison between our proposed method and CPEX for 40 node network

using our BPSO and CPEX are shown in Fig. 4 and 5. For the network with $N=30$, the schedule obtained in our methodology and CPLEX has $10(M)$ time slots and the utilization of the whole network $(\rho)$ is $0.123(37 / 300)$. The same result is obtained in earlier studies [1], [9], [11]. For this $N=30$ network, the results obtained in an earlier study [14], are $M=10$ and $\rho=0.12$. In other studies [8], [12], the corresponding values are shown in Table II. For the network with $N=40$, the schedule obtained in our methodology has $8(M)$ time slots and the utilization of the whole network $(\rho)$ is 0.213 (68/320). The optimal solution using CPLEX has 8 time slots and the utilization of the whole network $(\rho)$ is $0.216(69 / 320)$. For this case, our methodology produces a schedule that has better $\rho$ (utilization of the whole network) compared to other studies [1], [9], [8], [11], [12]. The number of time slots $M$ obtained is the same $(M=8)$ in earlier studies [1], [9], [10], [11], [12], [14], but the value of $\rho$ obtained is less than 0.213 . This shows our methodology performs better than earlier studies. The results for $N=30$ and 40 are shown in Table II.

Now, we consider a large-scale network with $N=100$. We consider a 100 node network with 200 and 300 links. The TDMA schedule obtained using our BPSO are shown in Fig. 6 and 7. For the network with $N=100$ and 200 
Table II

COMPARISON OF OUR RESULTS FOR $N=30$ AND 40 NODE NETWORK: BASED ON NUMBER OF TIME SLOTS $M$ AND UTILIZATION $\rho$

\begin{tabular}{|c|c|c|c|c|}
\hline \multirow{3}{*}{ Algorithm } & \multicolumn{4}{|c|}{ Node } \\
\hline & \multicolumn{2}{|c|}{30} & \multicolumn{2}{|c|}{40} \\
\hline & $M$ & $\rho$ & $M$ & $\rho$ \\
\hline CPLEX & 10 & 0.123 & 8 & 0.216 \\
\hline Proposed method & 10 & 0.123 & 8 & 0.213 \\
\hline Chen[9] & 10 & 0.123 & 8 & 0.203 \\
\hline Gunasekaran[14] & 10 & 0.12 & 8 & 0.203 \\
\hline $\operatorname{Kim}[1]$ & 10 & 0.123 & 8 & 0.213 \\
\hline Wang[8] & 12 & 0.108 & 9 & 0.197 \\
\hline Wang[10] & 10 & N/A & 8 & N/A \\
\hline $\mathrm{Wu}[11]$ & 10 & 0.123 & 8 & 0.200 \\
\hline Yeo[12] & 11 & 0.112 & 8 & 0.188 \\
\hline
\end{tabular}

links, the schedule obtained in our methodology has $9(M)$ time slots and the utilization of the whole network $(\rho)$ is 0.161 (145/900). The optimal solution using CPLEX has 9 time slots and the utilization of the whole network $(\rho)$ is 0.163 (147/900). For this case, our methodology produces a schedule that has better $\rho$ (utilization of the whole network) compared to other studies [1], [6], [11]. The number of time slots $M$ obtained is the same $(M=9)$ in earlier studies [1], [6], [11], but the value of $\rho$ obtained is 0.161 . This shows our methodology performs better than the earlier studies.

For the case of a network with $N=100$ and 300 links, the schedule obtained in our methodology has $10(M)$ time slots and the utilization of the whole network $(\rho)$ is $0.115(115 / 1000)$. The optimal solution using CPLEX has 9 time slots and the utilization of the whole network $(\rho)$ is 0.112 (101/900). For this case, our methodology produces a schedule that has better $\rho$ (utilization of the whole network) compared to other studies [1], [6], [11]. The number of time slots obtained $M=10$ [1] and $M=11$ [6], [11] in earlier studies. This also shows that our methodology performs better than the earlier studies. The results obtained for $N=100$, with 200 and 300 links are shown in Table III.

We would like to mention here that this BPSO does not guarantee that we obtain the optimal solution for a given network. However, in our simulation results we are able to obtain better TDMA schedules (using BPSO) than the TDMA schedules obtained in earlier studies [1], [6], [9], [8], [10], [11], [12], [14]. We can also find the best solution close to the optimal solution within limited computation time using BPSO while CPLEX needs much longer computation time to find the optimal solution.

\section{CONCLUSiON}

The broadcast scheduling problem (BSP) is considered with the objective of obtaining the best/optimal TDMA schedule. This schedule is to minimize the number of time slots $(M)$ and maximize the utilization $(\rho)$. This is known as BSP and shown as an NP-complete problem in

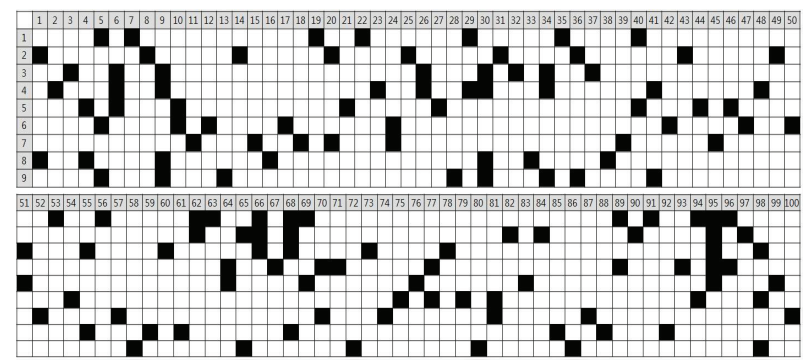

(a) The best solution using our proposed method

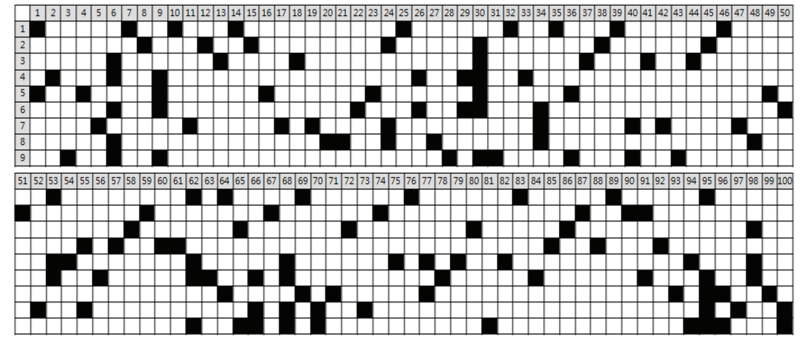

(b) The optimal solution using CPLEX

Figure 6. Comparison between our proposed method and CPEX for 100 nodes with 200 links

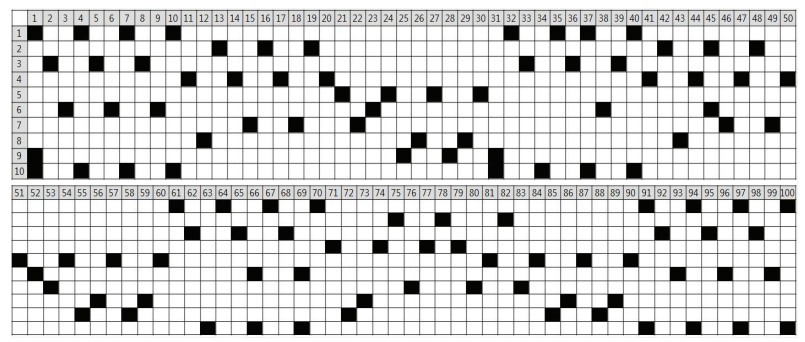

(a) The best solution using our proposed method

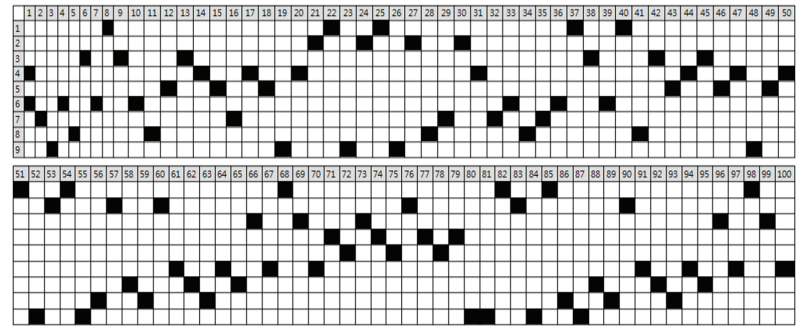

(b) The optimal solution using CPLEX

Figure 7. Comparison between our proposed method and CPEX for 100 nodes with 300 links

earlier studies. A BPSO for finding an optimum conflictfree transmission schedule for a broadcast radio network is presented to this NP-complete problem. Simulation results are presented for networks of size 15, 30, 40, and 100. Our results are compared with earlier methods such as genetic algorithms, mean field annealing or simulated annealing with valid solution mechanism, neural networks, factor graph and sum product algorithm, and sequential vertex coloring 
Table III

COMPARISON OF OUR RESULTS FOR $N=100$ LARGE-SCALE NETWORK: BASED ON NUMBER OF TIME SLOTS $M$ AND UTILIZATION $\rho$

\begin{tabular}{lcccc}
\hline \multirow{2}{*}{ Algorithm } & \multicolumn{4}{c}{ Node } \\
\cline { 2 - 5 } & \multicolumn{2}{c}{$100(200$ links $)$} & \multicolumn{1}{c}{$100(300$ links $)$} \\
\cline { 2 - 5 } & $M$ & $\rho$ & 9 & $\rho$ \\
\hline CPLEX & 9 & 0.163 & 10 & 0.112 \\
Proposed method & 9 & 0.161 & 11 & 0.104 \\
Chakraborty[6] & 9 & 0.148 & 10 & 0.11 \\
Kim[1] & 9 & 0.152 & 11 & 0.109 \\
Wu[11] & 9 & 0.151 & & \\
\hline
\end{tabular}

algorithm. It is shown that our BPSO is able to perform better than the earlier methods both in minimizing the number of time slots $(M)$ and maximizing utilization $(\rho)$. In our study, a valid solution mechanism is used in BPSO. We are able to achieve better results even for networks with 100 nodes using proposed BPSO in this paper. The results obtained using our methodology is compared with all the other earlier solution methods and CPLEX optimal solution, in terms of number of used time slots and utilization.

\section{ACKNOWLEDGMENT}

This work is supported partly by the National Natural Science Foundation of China (Grant No. 61173035), the Program for New Century Excellent Talents in University (Grant No. NCET-11-0861), the Fundamental Research Funds for the Central Universities (Grant No. 2012TD027).

\section{REFERENCES}

[1] G. Kim, S. Kim, I. Kim, D. Kim, V. Mani, and J. Moon, "An efficient simulated annealing with a valid solution mechanism for tdma brodcast scheduling problem," International Journal of innovative Computing Information and Control, vol. 7, no. 3, pp. 1181-1191, 2011.

[2] L. Davis and M. Mitchell, Handbook of genetic algorithms. Van Nostrand Reinhold, 1991.

[3] D. Goldberg, Genetic algorithms in search, optimization, and machine learning. Addison-wesley, 1989.

[4] J. Holland, Adaptation in natural and artificial systems. University of Michigan press, 1975, no. 53.

[5] Z. Michalewicz, Genetic algorithms+ data structures. Springer, 1996.

[6] G. Chakraborty, "Genetic algorithm to solve optimum tdma transmission schedule in broadcast packet radio networks," Communications, IEEE Transactions on, vol. 52, no. 5, pp. 765-777, 2004.

[7] S. Kirkpatrick, C. Gelatt Jr, and M. Vecchi, "Optimization by simulated annealing," science, vol. 220, no. 4598, pp. 671680, 1983.

[8] G. Wang and N. Ansari, "Optimal broadcast scheduling in packet radio networks using mean field annealing," Selected Areas in Communications, IEEE Journal on, vol. 15, no. 2, pp. 250-260, 1997.
[9] J. Chen, Y. Wang, and J. Chen, "A novel broadcast scheduling strategy using factor graphs and the sum-product algorithm," Wireless Communications, IEEE Transactions on, vol. 5, no. 6, pp. 1241-1249, 2006.

[10] L. Wang and H. Shi, "A gradual noisy chaotic neural network for solving the broadcast scheduling problem in packet radio networks," Neural Networks, IEEE Transactions on, vol. 17, no. 4, pp. 989-1000, 2006.

[11] X. Wu, B. Sharif, O. Hinton, and C. Tsimenidis, "Solving optimum tdma broadcast scheduling in mobile ad hoc networks: a competent permutation genetic algorithm approach," in Communications, IEE Proceedings-, vol. 152, no. 6. IET, 2005, pp. $780-788$.

[12] J. Yeo, H. Lee, and S. Kim, "An efficient broadcast scheduling algorithm for tdma ad-hoc networks," Computers \& operations research, vol. 29, no. 13, pp. 1793-1806, 2002.

[13] J. Kennedy and R. Eberhart, "A discrete binary version of the particle swarm algorithm," in Systems, Man, and Cybernetics, 1997. Computational Cybernetics and Simulation., 1997 IEEE International Conference on, vol. 5. IEEE, 1997, pp. 4104-4108.

[14] R. Gunasekaran, S. Siddharth, P. Krishnaraj, M. Kalaiarasan, and V. Rhymend Uthariaraj, "Efficient algorithms to solve broadcast scheduling problem in wimax mesh networks," Computer Communications, vol. 33, no. 11, pp. 1325-1333, 2010. 